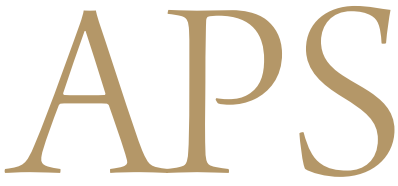

Archives of Plastic Surgery

\title{
Cognitive Investigation Study of Patients Admitted for Cosmetic Surgery: Information, Expectations, and Consent for Treatment
}

\author{
Mauro Barone ${ }^{1}$, Annalisa Cogliandro ${ }^{1}$, Giuseppe La Monaca ${ }^{2}$, Vittoradolfo Tambone ${ }^{2}$, \\ Paolo Persichetti ${ }^{1}$ \\ ${ }^{1}$ Plastic and Reconstructive Surgery Unit, Campus Bio-Medico University of Rome, Rome; ${ }^{2}$ Institute of Philosophy of Scientific and \\ Technological Activity, Campus Bio-Medico University of Rome, Rome, Italy
}

Background In all branches of medicine, it is the surgeon's responsibility to provide the patient with accurate information before surgery. This is especially important in cosmetic surgery because the surgeon must focus on the aesthetic results desired by the patient.

Methods An experimental protocol was developed based on an original questionnaire given to 72 patients. The nature of the responses, the patients' motivation and expectations, the degree of patient awareness regarding the planned operation, and the patients' perceptions of the purpose of the required consent for cosmetic surgery were all analyzed using Fisher's exact test.

Results Candidates for abdominal wall surgery had significantly more preoperative psychological problems than their counterparts did $(P=0.035)$. A significantly different percentage of patients under 40 years of age compared to those over 40 years of age searched for additional sources of information prior to the operation $(P=0.046)$. Only $30 \%$ of patients with a lower educational background stated that the preoperative information had been adequate, whereas $92 \%$ of subjects with secondary schooling or a postsecondary degree felt that the information was sufficient $(P=0.001)$. A statistically significant difference was also present between patients according to their educational background regarding expected improvements in their quality of life postoperatively $(P=0.008)$.

Conclusions This study suggests that patients require more attention in presurgical consultations and that clear communication should be prioritized to ensure that the surgeon understands the patient's expectations.

Keywords Informed consent / Cosmetic surgery / Quality of life / Aesthetic
Correspondence: Mauro Barone Plastic and Reconstructive Surgery Unit, Campus Bio-Medico University of Rome, Via Alvaro del Portillo, Rome 200-00128, Italy

Tel: +39-06-22541-1220 Fax: +39-06-22541-1936

E-mail: maurosabbarone@gmail.com

Received: 5 Jun $2014 \bullet$ Revised: 12 Oct $2014 \bullet$ Accepted: 31 Oct 2014

pISSN: 2234-6163 • elSSN: 2234-6171 • http://dx.doi.org/10.5999/aps.2015.42.1.46 • Arch Plast Surg 2015;42:46-51

No potential conflict of interest relevant to this article was reported.

\section{INTRODUCTION}

There has been a recent increase in the frequency of cosmetic surgery, corresponding with the tendency of modern society to evaluate individual well-being based on one's physical appearance. There is a continual search for beauty, which is not only considered an aesthetic ideal but also an expression of personal satisfaction [1] and a balance between personality and external 
appearance [2]. While the value of plastic surgery has never been doubted, the same cannot be said for cosmetic surgery. Hostility toward cosmetic surgery has been lessened by the emergence of new concepts of health. Previously, the World Health Organization defined health as the absence of illness, but subsequently defined health as physical, psychological, and social well-being. This reformed concept of health encompasses the subjective value that individuals place on themselves and their bodies [3].

This study aims to analyze the level of informedness and awareness among patients requesting cosmetic surgery using a questionnaire completed by patients before surgery. The questionnaire was created in collaboration between the Department of Plastic, Reconstructive and Cosmetic Surgery and the Institute of Philosophy of Scientific and Technological Activity of our hospital.

The foundation of cosmetic surgery is the differentiation between health and beauty. One may wonder where concepts of beauty are generated in modern culture. The ideals of beauty today are shaped outside the traditional channels of philosophy and art academies, and instead are influenced by the idealized images of beauty portrayed in fashion magazines and popular media. Advertising, tabloids, and works of fiction (e.g., novels, movies, and music videos) convey a specific vision of beauty. Important contributions have also come from literature, poetry, architecture, painting, theology, and mathematics. If medicine has not actively participated in this discussion, it must do so now if it does not intend to be reduced to mere technique [4]. Only through an approach that takes into account the complexity of the subject of investigation, human beauty, and the technical means used to achieve it, avoiding any kind of naive reduction, can "cooperating reduction" be achieved [5].

\section{METHODS}

An experimental protocol was developed based on the original questionnaire and was given to 72 patients. The nature of the responses, the patients' motivation and expectations, the degree of patient awareness regarding the planned operation, and the patients' perceptions of the purpose of the required consent for cosmetic surgery were all analyzed. The ethics committee of our hospital approved the questionnaire and authorized it to be administered to patients. The questionnaire was administered between August and December 2013.

\section{Questionnaire}

The questionnaire consisted of 17 multiple-response questions and two open-response questions; the data section was com- pleted by the surgeon, whereas the questionnaire itself was completed by the patient before surgery.

After statistically analyzing the answers to the multiple-response questions, it was possible to gather information relevant to the initial aims of our study. From the answers to the open-response questions, it was possible to analyze the patients' motives for undergoing cosmetic surgery.

\section{Sample selection}

Patients of Italian nationality who were older than 18 years of age and had requested cosmetic surgery were eligible for inclusion. However, psychiatric patients, anyone requesting reconstructive plastic surgery (postoncological treatment or posttrau$\mathrm{ma}$ ), and anyone with an alcohol or drug addiction were excluded from the study population.

\section{Questionnaire administration and data collection}

The questionnaire was conceived by the authors as a patient-administered test supplied by a medical interviewer to patients immediately after completing the surgical consent form and agreeing to surgery, but before undergoing surgical treatment.

\section{Characteristics of the sample}

The 72 patients consisted of 60 women and 12 men, with an average age of 40 years. Most had completed secondary school and belonged to categories 2, 4, and 8, according to the Italian National Institute of Statistics (ISTAT), which divides the population into nine categories. These categories are as follows: category 1 , legislators, managers, and entrepreneurs; category 2 , intellectual, scientific, and highly specialized professions; category 3, technical professions; category 4, employees; category 5, business and service professions; category 6 , artisans, and farmers; category 7, plant and semi-skilled workers of fixed and mobile machinery; category 8 , occupations; and category 9 , armed forces (Table 1).

\section{Surgical procedures}

Among the 72 patients undergoing surgery, rhinoplasty (20.8\%) and surgery in the face/neck area (45.8\%) accounted for most of the surgical procedures (Table 2). This corresponds to a recent study on aesthetic surgery, which highlighted both the nose and periorbital regions as the most common areas for surgery, especially in women [6].

\section{Data analyses}

All data obtained from the 72 questionnaires were collected in a single Microsoft Excel file and subjected to statistical analyses.

The results were evaluated by calculating the percentages of 
the patients' answers to various questions using the Fisher's exact test for $2 \times 2$ tables or by the Pearson chi-square test. Differences in the frequency of specific answers were considered statistically significant when $\mathrm{P}<0.05$.

\section{RESULTS}

The incidence of preoperative psychological problems according to the body area scheduled for treatment (i.e., face and neck, breast, abdomen, and buttocks/thighs) was examined. Preoper-

\begin{tabular}{|c|c|}
\hline \multicolumn{2}{|l|}{ Characteristic } \\
\hline \multicolumn{2}{|l|}{ Sample } \\
\hline Male & 12 \\
\hline Female & 60 \\
\hline \multicolumn{2}{|l|}{ Age (yr) } \\
\hline Male & $39(18-66)$ \\
\hline Female & $41(18-74)$ \\
\hline \multicolumn{2}{|l|}{ Country (n [\%]) } \\
\hline Northern Italy & $3(4.2)$ \\
\hline Central Italy & $66(91.7)$ \\
\hline Southern Italy & $3(4.2)$ \\
\hline \multicolumn{2}{|l|}{ Study qualification (n [\%]) } \\
\hline Lower study qualification & $10(13.9)$ \\
\hline Secondary school & $37(51.4)$ \\
\hline Graduates & $25(34.7)$ \\
\hline \multicolumn{2}{|c|}{ Classification of ISTAT professional categories } \\
\hline Categories 1-2 & 23 \\
\hline Categories 3-4-5-6-7-8-9 & 49 \\
\hline
\end{tabular}

\section{Table 2. Surgical procedures}

\begin{tabular}{|lc|}
\hline Surgical procedures & Number (\%) \\
\hline Rhinoplasty & $15(20.8)$ \\
Otoplasty & $2(2.8)$ \\
Blepharoplasty & $13(18)$ \\
Face lift & $3(4.2)$ \\
Breast augmentation & $8(11.1)$ \\
Mastopexy & $7(9.7)$ \\
Breast reduction & $4(5.6)$ \\
Thigh lift & $2(2.8)$ \\
Abdominoplasty & $5(7.0)$ \\
Liposuction & $13(18)$ \\
\hline
\end{tabular}

ative psychological problems were found in $66.7 \%$ of the candidates for abdominal wall surgery, which was significantly $(\mathrm{P}=$ 0.035 ) higher than that among patients receiving all other procedures (Table 3).

Patients were divided into two age groups ( $\geq 40$ years and $\leq 40$ years). The frequency of preoperative psychological problems was assessed according to age $(66.7 \%$ in those $<40$ years and $74.4 \%$ in patients $>40$ years). In addition, whether patients sought out additional sources of information during the preoperative period was analyzed. Almost all patients under 40 years of age (93.3\%) had searched for additional information regarding the surgical procedure, which was significantly higher than the percentage of those over $40(76.2 \%)$ who had sought out additional information $(\mathrm{P}=0.046)$ (Table 4$)$.

Expectations about improved quality of life after surgery were assessed according to the educational background of the patients. It was observed that the patients with a lower educational level had significantly higher expectations than the rest of the sample $(\mathrm{P}=0.020)$. Perceptions about the adequacy of the information received during the presurgical consultation were also analyzed. Only $30 \%$ of the patients with a lower educational background considered this information to be adequate, which reflected a statistically significant difference compared to patients with a higher educational background $(\mathrm{P}=0.001)$ (Table 5).

Cosmetic procedures were divided into major and minor in-

Table 3. Statistical analysis: anatomical areas

\begin{tabular}{|lccc|}
\hline & Abdomen & Other anatomical area & P-value \\
\hline $\begin{array}{l}\text { Psychological } \\
\text { problem (\%) }\end{array}$ & 66.7 & 25.4 & 0.035 \\
\hline $\begin{array}{l}\text { Statistically significant result, } \\
\text { P-value } \geq 0.05 .\end{array}$ \\
\hline
\end{tabular}

Table 4. Statistical analysis: age evaluations

\begin{tabular}{|lccl|}
\hline Age (yr) & Under $\mathbf{4 0}(\%)$ & Over $\mathbf{4 0}(\%)$ & P-value \\
\hline Psychological problem & 66.7 & 74.4 & 0.488 (NS) \\
$\begin{array}{l}\text { Using further information } \\
\text { sources }\end{array}$ & 93.3 & 76.2 & 0.046 (S) \\
\hline $\begin{array}{l}\text { Statistically significant result, P-value }<0.05 \\
\text { sult, } \text { P-value }>0.05 \text { (NS). non-statistically significant re- }\end{array}$ \\
\hline
\end{tabular}

\section{Table 5. Statistical analysis: study qualification}

\begin{tabular}{|lcccc}
\hline \multicolumn{1}{c}{ Study qualification } & \multicolumn{3}{c}{ Study qualification (\%) } & Upper \\
\cline { 2 - 4 } & Lower & Intermediate & 48 & P-value \\
\hline Patient expectations concerning quality of life & 90 & 75.8 & 92 & 0.020 (S) \\
Information received was considered adequate & 30 & 78.4 & & 0.001 (S) \\
\hline Statistically significant result, P-value $<0.05$ (S); non-statistically significant result, P-value $>0.05$ (NS). & \\
\hline
\end{tabular}


terventions according to the type of anesthesia, the length of the operation time, and the length of the patient's stay in the hospital. We then examined whether these two groups of patients had a different awareness of the surgical risks after informed consent was obtained; $89.3 \%$ of the candidates for minor cosmetic surgery considered themselves to be aware of the risks, whereas all patients requesting major surgery considered themselves to be aware of the risks $(\mathrm{P}=0.028)$. Moreover, we investigated whether the acquired information was considered adequate; $60.7 \%$ of patients undergoing major cosmetic surgery and $86.4 \%$ of patients undergoing minor procedures considered the information obtained to be sufficient $(\mathrm{P}=0.024)$ (Table 6).

After dividing the patients into high level (categories 1 and 2 of ISTAT) and low level (categories 3-9 of ISTAT) occupation groups, it was examined whether there were different expectations in improvements in their quality of life after cosmetic surgery. A statistically significant difference was found between the two groups $(\mathrm{P}=0.008)$. Postoperative improvements were expected by $48 \%$ of those in the high-level group, whereas $78.7 \%$ expected improvements in the low-level group (Table 7).

\section{DISCUSSION}

Cosmetic surgery is considered a means of improving a person's appearance. It is also used in cases of pathological necessity [7]. However, the results from our questionnaire highlight that there was a need for the cosmetic surgeons to improve the patients' quality of life and allow them to overcome difficulties related to psychological and social problems. Furthermore, the obtained data suggests that the level of information received by patients

\section{Table 6. Statistical analysis: surgical procedures}

\begin{tabular}{|lccc|}
\hline Surgical procedures & Major surgery & Minor surgery & P-value \\
\hline Awareness of risks & 100 & 89.3 & 0.028 (S) \\
Information received was & 60.7 & 86.4 & 0.024 (S) \\
considered adequate & & & \\
\hline
\end{tabular}

Statistically significant result, P-value $<0.05$ (S); non-statistically significant result, P-value $>0.05$ (NS). before surgery is inadequate in a significant number of cases. Whatever the branch of medicine, it is the surgeon's responsibility to inform the patient accurately prior to surgery and is especially important in cosmetic surgery since the surgeon must concentrate more on the aesthetic results desired by the patient. On the other hand, surgery is a drastic assault on biological tissue, and reactions to such procedures cannot always be foreseen. The majority of our study patients received their first cosmetic surgery from a plastic surgeon, and most complained about a physical problem that persisted for more than one year postoperatively. Patients considered for abdominoplasty represented $66.7 \%$ of the cases with psychological problems; these patients responded to the question, "Do you think that cosmetic surgery will improve your quality of life? If so, why?" by explaining that a pendulous abdomen or other factors related to abdominal problems resulted in great discomfort, not only from a functional point of view (e.g., during movement) but also in their daily life (e.g., putting on clothes). In fact, a recent study reported that abdominoplasty was the most influential factor in their patients' quality of life and daily relationships [8].

In addition, imperfections of the face, neck, breasts, buttocks, or thighs were considered physical problems that, according to our patients, required surgery to improve their bodily appearance. These problems are related to these patients' psychological well-being. In general, problems that influence a person to have plastic surgery therefore may change his/her perception of his/her quality of life in a specific way [9]. Furthermore, despite the level of expectation before surgery, the changes that occurred after the planned aesthetic surgery, the lifestyle led before surgery, and the degree of satisfaction with one's life overall are the most influential factors regarding post-surgical satisfaction. However, cosmetic surgery should not aim to change patients' lives because this expectation will always lead to dissatisfaction with the results [10].

Patients' expectations regarding improvements in their quality of life were divided by occupation group and revealed significant results. Patients employed in the low occupation group and with a lower educational background were more optimistic about their postcosmetic surgery results than their counterparts

Table 7. Statistical analysis: patient expectations in improvement of quality of life/Classification of ISTAT professional categories

\begin{tabular}{|c|c|}
\hline Classification of ISTAT professional categories & Categories 1-2 \\
\hline Patient expetations concerning quality of life (\%) & 48 \\
\hline
\end{tabular}


were. Only $48 \%$ of patients who had graduated or belonged to the high occupation group were optimistic about their quality of life after cosmetic surgery. These data appears to have been influenced by the patient's lifestyle, educational background, and occupation. Furthermore, the level of social/cultural awareness and presurgical expectations influence the degree of awareness and rational thinking about surgical treatments, and these factors add to the value and significance attributed to the surgery in question. Exaggerated presurgical expectations hinder cosmetic surgery since people from lower classes consider cosmetic surgery a means of obtaining personal satisfaction in their lives, if only to have the single aim of emulating another person or attaining a higher quality of life.

In the analysis stratified into two age groups, the sources of information sought by patients before their cosmetic surgery as well as the information supplied by the surgeon before surgery were significantly different between the two age groups. In patients under 40 years old, $93.3 \%$ turned to other sources to obtain further information, with the internet being the most common source. However, the reliability of the information available on the internet can critically influence the patient's expectations and sometimes lead to legal disputes between the surgeon and patient that are dictated by the patient's dissatisfaction with the results of the surgical procedure [11]. A common difficulty among cosmetic surgeons is convincing patients of the realistic objectives of the surgery and separating these objectives from the unrealistic expectations and unclear information obtained elsewhere. Most people in our study sought information from outside sources before consulting their surgeon to gain general information, to help them to ask appropriate questions, and express any doubts before requesting surgery [12].

Interestingly, only $30 \%$ of the patients with a lower educational background stated that the information they had received before surgery was adequate, whereas $92 \%$ of the patients who had completed secondary schooling or higher felt that the information provided before surgery was sufficient. This discrepancy appears to reflect a failure in communication between the surgeon and patient, which may negatively affect those with a lower educational background. This finding is of great interest because the pre-surgical information provided by the surgeon has the specific aim of allowing the patient to conscientiously exercise their right of therapeutic self-determination, independent of the degree of education or occupation.

The surgical procedures were divided into two categories: major or minor. The degree of satisfaction with presurgical information differed between patients in the two groups. Among patients who underwent minor surgery, $86 \%$ stated that the information they received was poor, whereas $60.7 \%$ of patients un- dergoing major surgery felt that the pre-surgical information was poor. These findings are of substantial importance in surgical cases with a high level of risk where surgery can cause permanent bodily changes with important consequences for the patient's life [13]. Therefore, the patient's need for presurgical information increases with the technical complexity of the planned surgical treatment.

However, insufficient information can lead to postoperative issues related to communication between the doctor and patient that can compromise their relationship and lead to legal action against the surgeon. In fact, a patient has a right to reproach their surgeon for removing his/her freedom of self-determination if the surgeon provides insufficient information.

Problems related to communicating information to patients who request treatment is, for the most part, a quite significant and unique part of cosmetic surgery because cosmetic surgery is more commonly undertaken to correct an external defect for aesthetic reasons than it is to correct a pathology for health and survival [14]. From this perspective, both the patient and doctor benefit from an accurate, documented, and informative process that preliminarily defines the aim of the operation and the realistic achievable results. Additionally, all risks and unforeseen complications should be clarified. By correctly informing the patient before obtaining consent for surgery, the surgeon may legally protect him/herself from possible medical negligence lawsuits.

\section{REFERENCES}

1. Persichetti P, Simone P, Tambone V. About beauty. Plast Reconstr Surg 2004;114:270-1.

2. Fusaschi M. Corporalmente corretto: note di antropologia. Roma: Meltemi; 2008.

3. Benicasa M. Liceità e fondamento dell'attività medico chirurgica a scopo terapeutico. Riv It Dir Proc Pen 1980:713e ss.

4. Persichetti P, Russo MT. Cosm-Etica: chirurgia estetica, corpo e bellelezza. Milano: Mc Graw-Hill; 2012.

5. Tambone V, Ghilardi G. Aware and cooperative reduction. Clin Ter 2012;163:e133-43.

6. Sezgin B, Findikcioglu K, Kaya B, et al. Mirror on the wall: a study of women's perception of facial features as they age. Aesthet Surg J 2012;32:421-5.

7. Ghigi R. Per piacere: storia culturale della chirurgia estetica. Bologna: Il mulino; 2008.

8. Papadopulos NA, Staffler V, Mirceva V, et al. Does abdominoplasty have a positive influence on quality of life, self-esteem, and emotional stability? Plast Reconstr Surg 2012; 129:957e-962e.

9. Papadopulos NA, Kovacs L, Krammer S, et al. Quality of life 
following aesthetic plastic surgery: a prospective study. J Plast Reconstr Aesthet Surg 2007;60:915-21.

10. Ozgur F, Tuncali D, Guler Gursu K. Life satisfaction, self-esteem, and body image: a psychosocial evaluation of aesthetic and reconstructive surgery candidates. Aesthetic Plast Surg 1998;22:412-9.

11. Alagoz MS, Basterzi AD, Uysal AC, et al. The psychiatric view of patients of aesthetic surgery: self-esteem, body image, and eating attitude. Aesthetic Plast Surg 2003;27:345-8.

12. Kamburoglu HO, Ozgur F. Postoperative satisfaction and the patient's body image, life satisfaction, and self-esteem: a retrospective study comparing adolescent girls and boys after cosmetic surgery. Aesthetic Plast Surg 2007;31:739-45.

13. von Soest T, Kvalem IL, Roald HE, et al. The effects of cosmetic surgery on body image, self-esteem, and psychological problems. J Plast Reconstr Aesthet Surg 2009;62:1238-44.

14. von Soest T, Kvalem IL, Skolleborg KC, et al. Psychosocial changes after cosmetic surgery: a 5-year follow-up study. Plast Reconstr Surg 2011;128:765-72. 